\title{
Computational Spectroscopy of the $\mathrm{Cr}-\mathrm{Cr}$ Bond in Coordination Complexes
}

\author{
Toru Shiozaki ${ }^{\dagger}$ and Bess Vlaisavljevich ${ }^{*, \hbar}$ \\ $†$ Quantum Simulation Technologies, Inc., Cambridge, MA 02139 \\ \$Department of Chemistry, University of South Dakota, Vermillion SD, 57069, USA \\ E-mail: bess.vlaisavljevich@usd.edu
}

\begin{abstract}
We report the accurate computational vibrational analysis of the $\mathrm{Cr}-\mathrm{Cr}$ bond in dichromium complexes using second-order multireference complete active space methods (CASPT2), allowing direct comparison with experimental spectroscopic data both to facilitate interpreting the low-energy region of the spectra and to provide insights into the nature of the bonds themselves. Recent technological development by the authors has realized such computation for the first time. Accurate simulation of the vibrational structure of these compounds has been hampered by their notorious multiconfigurational electronic structure that yields bond distances that do not correlate with bond order. Some measured $\mathrm{Cr}-\mathrm{Cr}$ vibrational stretching modes, $v\left(\mathrm{Cr}_{2}\right)$, have suggested weaker bonding, even for so-called ultrashort $\mathrm{Cr}-\mathrm{Cr}$ bonds, while others are in line with the bond distance. Here we optimize the geometries and compute $v\left(\mathrm{Cr}_{2}\right)$ with CASPT2 for three well-characterized complexes, $\mathrm{Cr}_{2}\left(\mathrm{O}_{2} \mathrm{CCH}_{3}\right)_{4}\left(\mathrm{H}_{2} \mathrm{O}\right)_{2}, \mathrm{Cr}_{2}(\mathrm{mhp})_{4}$, and $\mathrm{Cr}_{2}(\mathrm{dmp})_{4}$. We obtain CASPT2 harmonic $v\left(\mathrm{Cr}_{2}\right)$ modes in good agreement with experiment at $282 \mathrm{~cm}^{-1}$ for $\mathrm{Cr}_{2}(\mathrm{mhp})_{4}$ and $353 \mathrm{~cm}^{-1}$ for $\mathrm{Cr}_{2}(\mathrm{dmp})_{4}$, compute ${ }^{50} \mathrm{Cr}$ and ${ }^{54} \mathrm{Cr}$ isotope shifts, and demonstrate that the use of the so-called IPEA shift leads to improved $\mathrm{Cr}-\mathrm{Cr}$ distances. Additionally, normal mode sampling was used to estimate anharmonicity along $v\left(\mathrm{Cr}_{2}\right)$ leading to an anharmonic mode of $272 \mathrm{~cm}^{-1}$ for $\mathrm{Cr}_{2}(\mathrm{mhp})_{4}$ and $333 \mathrm{~cm}^{-1}$ for $\mathrm{Cr}_{2}(\mathrm{dmp})_{4}$.
\end{abstract}




\section{Introduction}

The concept of multiple bonding between metals dates back to the late 1920s with the first structural confirmation in $1964 .{ }^{1}$ Over the last 60 years, multiple bonds between a wide variety of transition metals have been assigned bond orders ranging from single to sextuple. ${ }^{2-4}$ Nowadays, synthetic chemists seek out new metal-metal bonds for as catalysts, building blocks in metalorganic frameworks, photosensitizers, and molecular conductors. ${ }^{3,5-8}$ Group 6 metal-metal bonds have been broadly studied for their interesting bonding and unique photophysical properties. ${ }^{9,10}$ Of these, $\mathrm{Cr}-\mathrm{Cr}$ multiple bonds are notable because of their rich electronic structure and unique spectroscopic properties. Starting with Cotton's work in the 1970s, a variety of dichromium complexes have been synthesized; characterization by diffraction has shown that the bond distances range from 1.7 to $2.3 \AA .^{4,11-18}$ Although in the early days formal bond orders were estimated based on distances, computational work using multiconfigurational methods has established that the electronic structure of these complexes is multiconfigurational due to the small energy splitting between the $\sigma, \pi$, and $\delta$ orbitals. The concept of an effective bond order (EBO), that accounts for partial occupation of low-lying antibonding orbitals, has been used to quantify the nature of the overall $\mathrm{Cr}-\mathrm{Cr}$ bond that is composed of multiple partial bonds. ${ }^{19,20}$ Furthermore, these computations highlight that the multiconfigurational electronic structure of $\mathrm{Cr}_{2}$ bonds is more nuanced compared to $\mathrm{Mo}_{2}$ and $\mathrm{W}_{2}$ analogues. ${ }^{20}$ As a result, the $\mathrm{Cr}-\mathrm{Cr}$ bond order often does not correlate with bond distance. ${ }^{19-22}$ Note that for some particularly short $\mathrm{Cr}-\mathrm{Cr}$ distances, density functional theory (DFT) has been used successfully since the electronic structure can be represented by fully occupied bonding orbitals. ${ }^{23}$

The lack of correlation between bond distance and bond order motivated a number of vibrational studies of these compounds; diffraction studies alone cannot be used to predict bonding or, in turn, vibrational force constants. Da Re et al. emphasize the unique challenges in dichromium complexes by comparing the empirical force constants and bond distances for first and second row transition metals. For example, Mo(II)-Mo(II) bond distances empirically correlate to their vibrational frequencies, as expected, with force constants increasing monotonically with decreas- 
ing bond distance. ${ }^{24} \mathrm{Cr}$ (II)-Cr(II) bonds deviate from the norm and have multiple modes in the low-frequency region of the spectra involving the $\mathrm{Cr}$ centers, making peak assignment challenging. The measured force constants in two complexes with ultrashort $\mathrm{Cr}-\mathrm{Cr}$ bonds, $\mathrm{Cr}_{2}(\mathrm{mhp})_{4}$ and $\mathrm{Cr}_{2}(\mathrm{dmp})_{4}$, drew our interest $(\mathrm{mhp}=$ deprotonated 6-methyl-2-hydroxypyridine and $\mathrm{dmp}=2,6-$ dimethoxyphenyl). In the former, the $\mathrm{Cr}-\mathrm{Cr}$ stretching mode, $v\left(\mathrm{Cr}_{2}\right)$, was first assigned at 556 $\mathrm{cm}^{-1}$ by Cotton et al. but was later revised to be at 340 (or 400 ) $\mathrm{cm}^{-1}$ by Manning and Trogler. ${ }^{11,25}$ The $\mathrm{Cr}-\mathrm{Cr}$ bond in $\mathrm{Cr}_{2}(\mathrm{dmp})_{4}$ is even shorter, but the ambiguity surrounding the vibrational mode remains. An estimation based on the aggregate isotope shift would lead to the assignment at 650 $\mathrm{cm}^{-1}$; however, an alternative assignment, based on the average of three low-frequency bands assigned to include motion in the $\mathrm{Cr}-\mathrm{Cr}$ internal coordinate, would put $v\left(\mathrm{Cr}_{2}\right)$ closer to $365 \mathrm{~cm}^{-1} .^{24}$ In both complexes, the larger value is consistent with the force constant that would be predicted from a simple harmonic approximation based on the bond distance. The lower values would suggest a significantly weaker bond than one would expect from a formally quadruple bond.

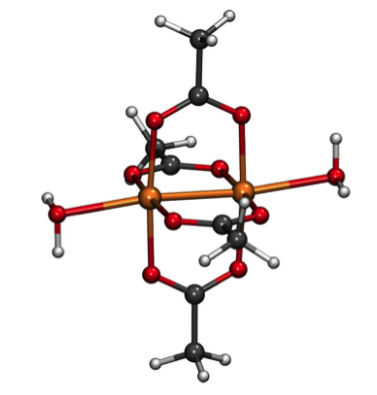

$\left[\mathrm{Cr}_{2}\left(\mathrm{O}_{2} \mathrm{CCH}_{3}\right)_{4}\right]\left(\mathrm{H}_{2} \mathrm{O}\right)_{2}$

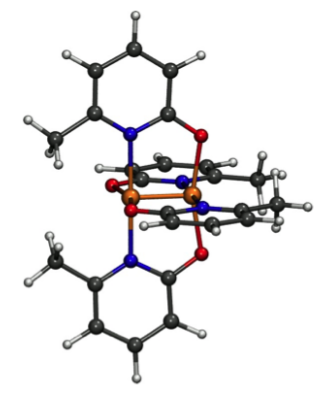

$\mathrm{Cr}_{2}(\mathrm{mhp})_{4}$

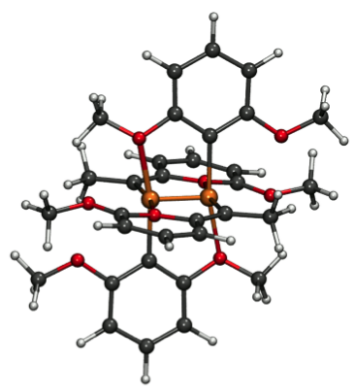

$\mathrm{Cr}_{2}(\mathrm{dmp})_{4}$

Figure 1: The dichromium complexes studied in this work. mhp $=$ deprotonated 6-methyl-2hydroxypyridine and $\mathrm{dmp}=2,6$-dimethoxyphenyl. $\mathrm{Cr}$ in orange, $\mathrm{N}$ in blue, $\mathrm{O}$ in red, $\mathrm{C}$ in gray, and $\mathrm{H}$ in white.

Vibrational analysis of compounds consisting of a few dozen atoms is routinely performed by DFT. However, as mentioned earlier, the electronic structure of dichromium complexes is highly multiconfigurational, which DFT cannot describe properly. ${ }^{19}$ For the computation of bond length, several procedures have been proposed to empirically correct DFT simulations. For example, DFT geometry optimizations can be performed keeping the $\mathrm{Cr}-\mathrm{Cr}$ bond distance fixed at the experi- 
mental value with subsequent analysis of the electronic structure with CASPT2. ${ }^{15}$ Alternatively, a series of constrained DFT optimizations can be performed to determine the $\mathrm{Cr}-\mathrm{Cr}$ bond distance point-wise with CASPT2 yielding good agreement with experiment. ${ }^{19-22}$ These hybrid approaches, however, are not applicable to the computation of the vibrational spectra. Moreover, full CASPT2 geometry optimization for complexes with transition metals had only been performed on small molecules, and even then not routinely. ${ }^{26-28}$

Here, we directly apply the CASPT2 electronic structure method to the prediction of vibrational spectra of $\mathrm{Cr}-\mathrm{Cr}$ complexes, in which the vibrational frequencies and normal modes are computed from the second derivative of CASPT2 energies. Full geometry optimizations with the CASPT2 method and subsequent harmonic vibrational analysis are made possible by utilizing the recently developed CASPT2 analytical gradient program and extending it to compute second-order energy derivatives by means of finite difference of the first derivatives. Anharmonic effects are known to be important from experimental work on these complexes; ${ }^{24}$ therefore, the anharmonicity of the $\mathrm{Cr}-\mathrm{Cr}$ harmonic stretching mode is studied.

We present the results for $\left[\mathrm{Cr}_{2}\left(\mathrm{O}_{2} \mathrm{CCH}_{3}\right)_{4}\right]\left(\mathrm{H}_{2} \mathrm{O}\right)_{2}, \mathrm{Cr}_{2}(\mathrm{mhp})_{4}$, and $\mathrm{C}_{2}(\mathrm{dmp})_{4}$ where mhp $=$ deprotonated 6-methyl-2-hydroxypyridine and $\mathrm{dmp}=2,6$-dimethoxyphenyl (Figure 1). The resulting vibrational frequencies are analyzed and comparisons with experimentally assigned $v\left(\mathrm{Cr}_{2}\right)$ peaks are made, providing insights into the nature of the $\mathrm{Cr}(\mathrm{II})-\mathrm{Cr}(\mathrm{II})$ bond. ${ }^{29}$

\section{Computational Details}

\section{Software Program for CASPT2 vibrational spectra}

The CASPT2 second-order energy derivatives are computed by the finite difference of the firstorder derivatives calculated using the analytical nuclear gradient program recently developed by the authors. ${ }^{27,28}$ We note in passing that the use of the analytical gradient program is essential in realizing the CASPT2 vibrational analysis because it reduces the computational cost by two- to three-orders of magnitude for the molecules in this study. An embarrassingly parallel algorithm 
has been included that allows the user to select the number of MPI processes required for each gradient calculation and distribute the $6 \mathrm{~N}$ displacements among different tasks. The Hessian is symmetrized, mass weighted, and the translational and rotational degrees of freedom are set to zero using the usual projection (see Ref. 30 for a concise summary). The normal modes are obtained by diagonalizing the projected mass-weighted Hessian yielding the normal modes (eigenvectors), and the corresponding harmonic vibrational frequencies can be calculated from the eigenvalues. The infrared intensity is also computed from the transition dipole moments. Raman intensities are not yet implemented.

In some of the calculations herein, we used Partial Harmonic Vibrational Analysis (PHVA) as described first by Head and coworkers ${ }^{31-35}$ and later by Li and Jensen ${ }^{30}$ to reduce the computational cost. In PHVA, a subblock of the Hessian matrix is diagonalized to obtained vibrational frequencies within that portion of the molecule. This scheme is particularly useful in this study because the $\mathrm{Cr}-\mathrm{Cr}$ stretching mode is localized, allowing us to skip some of the displacements, especially those associated with bulky ligands, that do not couple directly or indirectly with the $\mathrm{Cr}-\mathrm{Cr}$ stretching mode. The selection scheme of the atoms to be displaced is detailed below.

\section{Simulation Parameters}

CASPT2 geometry optimizations and vibrational frequencies have been performed as implemented in the BAGEL program package. ${ }^{36}$ An $(8 e, 8 o)$ active space is used consisting of the $3 d \sigma, \pi$, and $\delta$ bonding and antibonding orbitals, resulting from the interaction of the $3 d^{4}$ atomic configurations of the two Cr(II) centers (Figure 2 and 3, Figure S1).

For $\left[\mathrm{Cr}_{2}\left(\mathrm{O}_{2} \mathrm{CCH}_{3}\right)_{4}\right]\left(\mathrm{H}_{2} \mathrm{O}\right)_{2}$, computations were performed with cc-pVTZ on all atoms. ${ }^{37-39}$ See Table S5 for calculations using other basis sets. A real shift of 0.3 a.u. was used. ${ }^{28}$ The computation of harmonic vibrational frequencies with both the Full Harmonic Vibrational Approximation (FHVA) and PHVA was performed. Complete active space self-consistent field (CASSCF) geometry optimizations were conducted for comparison. For $\mathrm{Cr}_{2}(\mathrm{mhp})_{4}$ and $\mathrm{Cr}_{2}(\mathrm{dmp})_{4}$, CASPT2 geometry optimizations of the ground state followed by PHVA were performed using a basis set 
with cc-pVTZ on $\mathrm{Cr}$ and the first coordination sphere while cc-pVDZ was used on all other atom types. A real shift of 0.4 a.u. was used.

The CASPT2 energy was converged to $1 \times 10^{-8}$ and the forces were converged to $3 \times 10^{-4}$. The def2-TZVPP-JKFIT basis was used in all calculations for density fitting ${ }^{40}$ and the so-called IPEA shift was not employed since it is not yet implemented for CASPT2 nuclear gradients. ${ }^{41}$ In PHVA, the $\mathrm{Cr}$ atoms and the first coordination sphere were displaced.

A scan of the potential energy surface (PES) was then performed by displacing the geometry along the harmonic normal mode $\left(Q_{i}\right)$ prior to mass weighting in Cartesian increments of 0.25 for $\mathrm{Cr}_{2}(\mathrm{mhp})_{4}$ and and $\mathrm{Cr}_{2}(\mathrm{dmp})_{4}$. Single point CASPT2 calculations are performed using the same level of theory previously described. Coordinates are provided as supporting information for each displacement. Since there is literature precedent that the $\mathrm{Cr}-\mathrm{Cr}$ bond distance is affected by the choice of zeroth-order Hamiltonian, ${ }^{41-44}$ analogous CASPT2 calculations were performed along the normal mode using the OpenMolcas program package. An imaginary shift of 0.2 a.u. and the so-called IPEA shift of 0.25 was employed. ${ }^{41}$ ANO-RCC basis sets were used (see caption of Figures S2 and S3 for specific contractions).

DFT geometry optimizations and harmonic vibrational frequencies were computed as implemented in the Turbomole program package. ${ }^{45}$ The M06 functional ${ }^{46}$ was used with the def2-TZVP basis set. ${ }^{47}$ Singlet and triplet spin states were considered. Attempts to optimize the geometry with a broken-symmetry singlet routinely relax to the closed-shell singlet.

\section{Results and Discussion}

\section{CASPT2 Geometries}

Although DFT geometries are typically in very good agreement with experiment for transition metal complexes, the $\mathrm{Cr}-\mathrm{Cr}$ multiple bond presents particularly challenging electronic structure that leads to stark differences between DFT geometries and diffraction measurements. For these three complexes, the $\mathrm{Cr}-\mathrm{Cr}$ distances obtained by DFT deviate from diffraction by 0.151 to 0.641 
$\AA$ for the ground state singlet (Table 1). DFT severely underestimates the $\mathrm{Cr}-\mathrm{Cr}$ bond distance, because the electronic state is not described properly and is not a result of functional choice. The Cr-Cr distance in the M06 triplet geometry ranges from 1.922 to $2.497 \AA$ for the three complexes (Table S1)

Table 1: M06, CASPT2, and experimental Cr-Cr bond distances in $\AA$ are compared.

\begin{tabular}{llll}
\hline Complex & M06 & CASPT2 & Exp. \\
\hline$\left[\mathrm{Cr}_{2}\left(\mathrm{O}_{2} \mathrm{CCH}_{3}\right)_{4}\right]\left(\mathrm{H}_{2} \mathrm{O}\right)_{2}$ & 1.721 & 2.419 & $2.362^{[a]}$ \\
$\mathrm{Cr}_{2}(\mathrm{mhp})_{4}$ & 1.704 & 1.989 & $1.889(1)^{[b]}$ \\
$\mathrm{Cr}_{2}(\mathrm{dmp})_{4}$ & 1.698 & 1.922 & $1.849(2)^{[c]}$ \\
\hline
\end{tabular}

[a] $\operatorname{Ref} 48,[b] \operatorname{Ref} 11$, [c] $\operatorname{Ref} 49$

The comparison between CASPT2 gas phase geometries and experiment in $\mathrm{Cr}_{2}\left(\mathrm{O}_{2} \mathrm{CCH}_{3}\right)_{4}$ $\left(\mathrm{H}_{2} \mathrm{O}\right)_{2}$ is not straightforward due to the presence of hydrogen bonding between the apical $\mathrm{H}_{2} \mathrm{O}$ ligands in the solid-state structure. Nevertheless, qualitative comparisons can be made. This complex contains fewer atoms and its vibrational spectrum has been measured. Therefore, $\mathrm{Cr}_{2}\left(\mathrm{O}_{2} \mathrm{CCH}_{3}\right)_{4}$ $\left(\mathrm{H}_{2} \mathrm{O}\right)_{2}$ is presented to demonstrate the effect of basis set choice, to compare DFT, CASSCF, and CASPT2 geometries, and to compare CASPT2 $\mathrm{Cr}-\mathrm{Cr}$ stretching modes computed with PHVA to those from FHVA. While DFT underestimates the bond distances in the singlet state for $\mathrm{Cr}_{2}\left(\mathrm{O}_{2} \mathrm{CCH}_{3}\right)_{4}$ $\left(\mathrm{H}_{2} \mathrm{O}\right)_{2}$ by $0.650 \AA$ compared to experiment, the CASSCF distance is overestimated by $0.408 \AA$. CASPT2 reduces this overestimation to $0.057 \AA$ and a $\mathrm{Cr}-\mathrm{Cr}$ distance of $2.419 \AA$ is obtained. This is analogous to the overestimation of bond distances observed in Hartree-Fock which can be corrected by performing MP2 calculations.

CASPT2 geometries can be optimized for larger complexes containing metal-metal bonds (Table 1). $\mathrm{Cr}_{2}(\mathrm{mhp})_{4}$ and $\mathrm{Cr}_{2}(\mathrm{dmp})_{4}$ contain 58 and 79 atoms, respectively. Both complexes have been characterized by diffraction to yield $\mathrm{Cr}-\mathrm{Cr}$ distances of $1.889(1)$ and $1.849(2) \AA{ }^{11,12}$ These distances fall in the range of ultrashort, formally quadruple, $\mathrm{Cr}(\mathrm{II})-\mathrm{Cr}(\mathrm{II})$ bonds. As in $\mathrm{Cr}_{2}\left(\mathrm{O}_{2} \mathrm{CCH}_{3}\right)_{4}\left(\mathrm{H}_{2} \mathrm{O}\right)_{2}$, CASPT2 $\mathrm{Cr}-\mathrm{Cr}$ distances are in much better agreement with experiment for $\mathrm{Cr}_{2}(\mathrm{mhp})_{4}$ and $\mathrm{Cr}_{2}(\mathrm{dmp})_{4}$ with values of 1.989 and $1.922 \AA$, respectively; the calculated values are overestimated by 0.100 and $0.075 \AA$. 
Although Cr-ligand bond distances are in good agreement with experiment (Table S2), the difference between the computed and measured $\mathrm{Cr}-\mathrm{Cr}$ bond distances are larger than what can be considered excellent agreement despite the clear improvement over DFT. Ruipérez et al. studied diatomic $\mathrm{Cr}_{2}$ and showed that larger IPEA shifts resulted in shorter $\mathrm{Cr}-\mathrm{Cr}$ distances. ${ }^{42}$ The effect of the choice of zeroth-order Hamiltonian in these complexes is presented vida infra.

\section{Electronic Structure of the Cr-Cr Multiple Bond}

The large differences between the DFT and CASPT2 $\mathrm{Cr}-\mathrm{Cr}$ bond distances are a direct result of multiconfigurational character. The occupation numbers (ONs) associated with the CASSCF active orbitals for the complexes with the longest and shortest $\mathrm{Cr}-\mathrm{Cr}$ bonds, $\left[\mathrm{Cr}_{2}\left(\mathrm{O}_{2} \mathrm{CCH}_{3}\right)_{4}\right]\left(\mathrm{H}_{2} \mathrm{O}\right)_{2}$ and $\mathrm{Cr}_{2}(\mathrm{dmp})_{4}$, demonstrate this as they deviate significantly from $2.00,1.00$, or 0.00 (indicative of doubly, singly, or unoccupied orbitals, respectively) (Figures 2 and 3). Moreover, the largest contribution to the CASSCF wavefunction for $\left[\mathrm{Cr}_{2}\left(\mathrm{O}_{2} \mathrm{CCH}_{3}\right)_{4}\right]\left(\mathrm{H}_{2} \mathrm{O}\right)_{2}$ is the $\sigma^{2} \pi^{4} \delta^{2}$ configuration with only a $6.4 \%$ weight (Table 2). The $\sigma^{2} \pi^{4} \delta^{* 2}$ configuration follows with a contribution of $5.2 \%$, while all other configurations contribute less than $4 \%$. Furthermore, the effective bond order (EBO),

$$
E B O=\frac{\sum O N_{\text {bonding }}-\sum O N_{\text {antibonding }}}{2}
$$

is 0.714 (Table S3) for $\left[\mathrm{Cr}_{2}\left(\mathrm{O}_{2} \mathrm{CCH}_{3}\right)_{4}\right]\left(\mathrm{H}_{2} \mathrm{O}\right)_{2}$. The bond involves partial $\sigma, \pi$, and $\delta$ bonds where the antibonding orbitals are sufficiently populated resulting in a net bond order corresponding to a single bond and not a quadruple bond. $\mathrm{Cr}_{2}(\mathrm{mhp})_{4}$ and $\mathrm{Cr}_{2}(\mathrm{dmp})_{4}$ have slightly stronger bonds with EBOs of 1.863 and 2.202, still far weaker than the formal quadruple bond suggested based solely on bond distance. This is also reflected in the increased contribution of the $\sigma^{2} \pi^{4} \delta^{2}$ configuration to the ground state of 26.5 or $35.6 \%$ (Table 2). Finally, higher energy spin states (triplet and quintet) were computed at the CASPT2 ground state geometry (Table S4). The triplet is closest in energy by 11.2 and $13.3 \mathrm{kcal} / \mathrm{mol}$ for $\mathrm{Cr}_{2}(\mathrm{mhp})_{4}$ and $\mathrm{C}_{2}(\mathrm{dmp})_{4}$, respectively. 

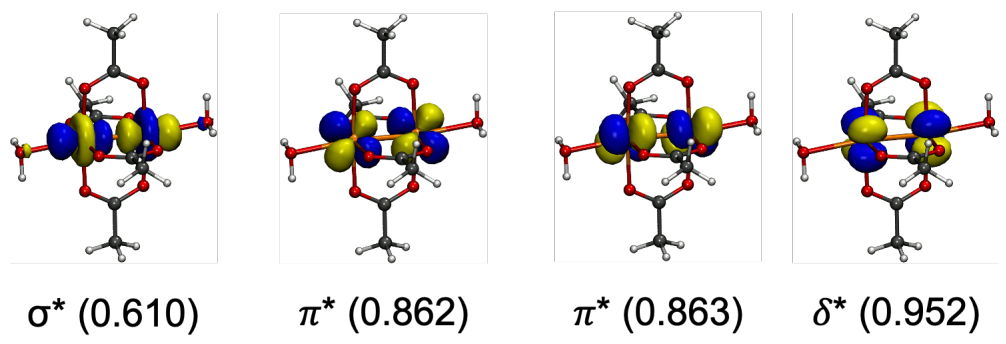

$\pi^{*}(0.862)$

$\pi^{*}(0.863)$

$\delta^{*}(0.952)$
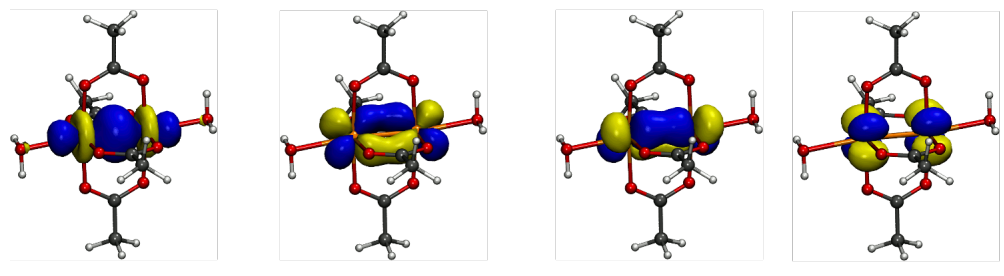

$\sigma(1.390)$

$\pi(1.138)$

$\pi(1.137)$

$\delta(1.048)$

Figure 2: CASSCF/cc-pVTZ active natural orbitals and occupation numbers for $\left[\mathrm{Cr}_{2}\left(\mathrm{O}_{2} \mathrm{CCH}_{3}\right)_{4}\right]\left(\mathrm{H}_{2} \mathrm{O}\right)_{2}$. An isovalue of 0.4 a.u. was used.
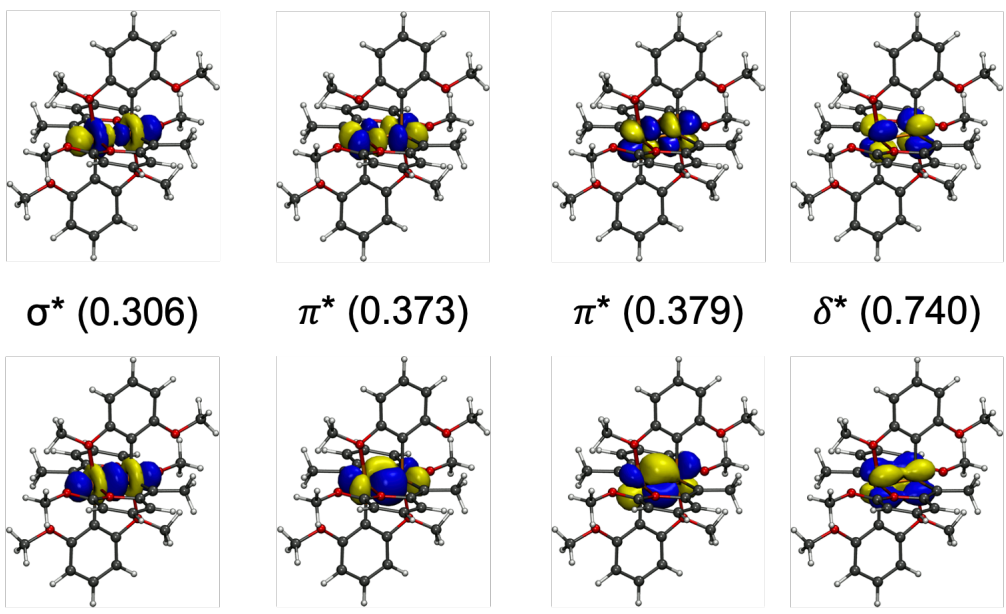

$\delta^{*}(0.740)$

$\sigma(1.693)$
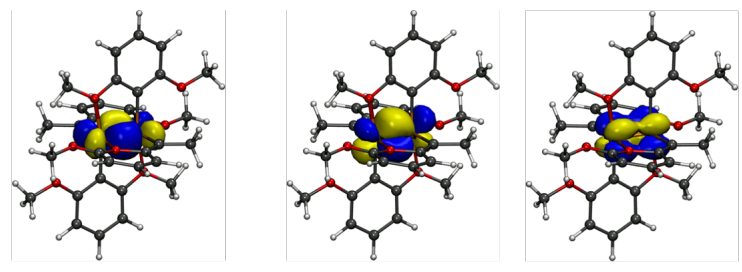

$\pi(1.628)$

$\pi(1.621) \quad \delta(1.260)$

Figure 3: CASSCF/cc-pVDZ,cc-pVTZ(Cr,O) active natural orbitals and occupation numbers for $\mathrm{Cr}_{2}(\mathrm{dmp})_{4}$. An isovalue of 0.4 a.u. was used. 
Table 2: Electronic configurations contributing greater than $5 \%$ to the total CASSCF wavefunction.

\begin{tabular}{lll}
\hline Complex & Dominant Configurations & Weight \\
\hline$\left[\mathrm{Cr}_{2}\left(\mathrm{O}_{2} \mathrm{CCH}_{3}\right)_{4}\right]\left(\mathrm{H}_{2} \mathrm{O}\right)_{2}$ & $\sigma^{2} \pi^{2} \pi^{2} \delta^{2} \delta^{* 0} \pi^{* 0} \pi^{* 0} \sigma^{* 0}$ & 6.4 \\
$\mathrm{Cr}_{2}(\mathrm{mhp})_{4}$ & $\sigma^{2} \pi^{2} \pi^{2} \delta^{0} \delta^{* 2} \pi^{* 0} \pi^{* 0} \sigma^{* 0}$ & 5.2 \\
& $\sigma^{2} \pi^{2} \pi^{2} \delta^{2} \delta^{* 0} \pi^{* 0} \pi^{* 0} \sigma^{* 0}$ & 26.5 \\
$\mathrm{Cr}_{2}(\mathrm{dmp})_{4}$ & $\sigma^{2} \pi^{2} \pi^{2} \delta^{0} \delta^{* 2} \pi^{* 0} \pi^{* 0} \sigma^{* 0}$ & 14.0 \\
& $\sigma^{2} \pi^{2} \pi^{2} \delta^{2} \delta^{* 0} \pi^{* 0} \pi^{* 0} \sigma^{* 0}$ & 35.4 \\
& $\sigma^{2} \pi^{2} \pi^{2} \delta^{0} \delta^{* 2} \pi^{* 0} \pi^{* 0} \sigma^{* 0}$ & 15.4 \\
\hline
\end{tabular}

\section{Harmonic Vibrational Analysis}

The $\mathrm{Cr}-\mathrm{Cr}$ symmetric stretching mode, $v\left(\mathrm{Cr}_{2}\right)$, was computed with FHVA for $\left[\mathrm{Cr}_{2}\left(\mathrm{O}_{2} \mathrm{CCH}_{3}\right)_{4}\right]\left(\mathrm{H}_{2} \mathrm{O}\right)_{2}$. The effect of the basis set is small, and PHVA yields similar performance but reduces the total computational cost significantly (Table S5). In $\mathrm{Cr}_{2}\left(\mathrm{O}_{2} \mathrm{CCH}_{3}\right)_{4}\left(\mathrm{H}_{2} \mathrm{O}\right)_{2}$, FHVA requires 216 gradient calculations for 36 atoms while displacing 12 atoms in PHVA requires 72 gradient calculations. Keeping in mind that hydrogen-bonding in the solid state makes comparison between CASPT2 and experiment qualitative, the electronic absorption spectrum suggests that the $\mathrm{Cr}-\mathrm{Cr}$ stretching mode lies between 150 and $250 \mathrm{~cm}^{-1} \cdot{ }^{50}$ FHVA CASPT2 predicts two modes with $v\left(\mathrm{Cr}_{2}\right)$ character at 162 and $276 \mathrm{~cm}^{-1}$. PHVA yields similar results with modes at 196 and $266 \mathrm{~cm}^{-1}$ (Table 3). On the other hand, DFT (M06) predicts this mode at $585 \mathrm{~cm}^{-1} v\left(\mathrm{Cr}_{2}\right)$, consistent with an unreasonably short $\mathrm{Cr}-\mathrm{Cr}$ distance.

Cotton et al. reported the Raman spectrum of $\mathrm{Cr}_{2}(\mathrm{mhp})_{4}$ and assigned the ground state $\mathrm{Cr}-$ Cr stretching mode at $556 \mathrm{~cm}^{-1} \cdot{ }^{11}$ At the time, this was the highest M-M stretching mode ever reported by a large margin and the $\mathrm{Cr}_{2}(\mathrm{dmp})_{4}$ complex, when measured, was expected to lead to an even higher value. This value is consistent with an empirically assigned force constant based on a simple diatomic oscillator approximation employing the measured $\mathrm{Cr}-\mathrm{Cr}$ distance. However, the vibronic features in the absorption and emission spectra as well as combined infrared and Raman analysis performed by Manning and Trogler ruled out this assignment suggesting that $v\left(\mathrm{Cr}_{2}\right)$ lies at a much smaller wavenumber, either 340 or $400 \mathrm{~cm}^{-1} \cdot{ }^{25}$ They specifically note that either of the two Raman bands could be the $\mathrm{Cr}-\mathrm{Cr}$ stretching mode but could not obtain solution spectra to 
further solidify the assignment. Since a vibronic progression at $320 \mathrm{~cm}^{-1}$, assigned to the so-called $\delta \rightarrow \delta^{*}$ excited state, was also observed, Manning and Trogler suggested that the $340 \mathrm{~cm}^{-1}$ peak was more likely to be the $\mathrm{Cr}-\mathrm{Cr}$ stretching mode. This was based on the observation of a reduction in $v\left(\mathrm{M}_{2}\right)$ by $10-15 \%$ in the excited state for quadruple bonded $\mathrm{M}_{2}$ systems.

The CASPT2 harmonic $\mathrm{Cr}-\mathrm{Cr}$ stretching mode is at $282 \mathrm{~cm}^{-1}$; however, modes at 266, 267, 359 , and $360 \mathrm{~cm}^{-1}$ consist of coupled metal-metal and metal-ligand stretching motions. We can compare $v\left(\mathrm{Cr}_{2}\right)$ with experiment directly but note that the average of all modes involving significant $\mathrm{Cr}-\mathrm{Cr}$ motion would give nearly the same value, $299 \mathrm{~cm}^{-1}$, supporting Manning and Trogler's assignment of $340 \mathrm{~cm}^{-1}$. Furthermore, these results reinforce previous conclusions based on the vibrational spectrum that the formally $\mathrm{Cr}_{2}$ quadruple bond in this complex is much weaker, consistent with the electronic structure (vida supra). Although, to our knowledge, isotopic labeling experiments have not been performed for $\mathrm{Cr}_{2}(\mathrm{mhp})_{4}, v\left(\mathrm{Cr}_{2}\right)$ shifts to $287 \mathrm{~cm}^{-1}$ for ${ }^{50} \mathrm{Cr}$ and 277 $\mathrm{cm}^{-1}$ for ${ }^{54} \mathrm{Cr}$. Similar sized shifts are observed for the modes consisting of combinations of metalmetal and metal-ligand motions (Table S6).

Additionally, $v\left(\mathrm{Cr}_{2}\right)$ was computed for $\mathrm{Cr}_{2}(\mathrm{dmp})_{4}$ and the predominate $\mathrm{Cr}-\mathrm{Cr}$ stretch is at 353 $\mathrm{cm}^{-1}$. The mode is the largest of the three complexes, consistent with having the shortest bond distance and highest bond order, but remains significantly smaller than empirical estimates. The CASPT2 value for $v\left(\mathrm{Cr}_{2}\right)$ is in good agreement with the estimate of this mode at $365 \mathrm{~cm}^{-1}$ based on averaging three bands observed in experiment. Specifically, three bands appear in experiments using the naturally occurring isotope $\left(\sim 87 \%{ }^{52} \mathrm{Cr}\right)$ at 345,363 , and $387 \mathrm{~cm}^{-1}$ (labeled as bands $\mathrm{a}, \mathrm{b}$, and c; Table 3) and were assigned to involve Cr motions due to their shifts upon isotope substitution. ${ }^{24}$ Since several CASPT2 normal modes contributions from $\mathrm{Cr}-\mathrm{Cr}, \mathrm{Cr}-\mathrm{C}$ and $\mathrm{Cr}-\mathrm{N}$ stretching motions, further analysis including ${ }^{50} \mathrm{Cr}$ and ${ }^{54} \mathrm{Cr}$ isotopes was performed (Table S7). Of the normal modes computed by CASPT2 with mixed metal-metal and metal-ligand stretching motions, the two at 310 and $324 \mathrm{~cm}^{-1}$ have the largest shifts upon isotope substitution (Table S6). We tentatively assign these two modes along with the relatively unmixed $v\left(\mathrm{Cr}_{2}\right)$ mode as bands a, b, and c (Table 3). The CASPT2 bands are shifted to lower wavenumbers at 310, 324, and 353 
$\mathrm{cm}^{-1}$ but the spacing between peaks is in good agreement with experiment. This is likely due in part to the overestimation of the calculated bond distance.

This assignment is further supported by the calculated isotope shifts. The predicted shift due to substituting ${ }^{54} \mathrm{Cr}$ with ${ }^{50} \mathrm{Cr}$ for a simple diatomic oscillator with a mode of $340 \mathrm{~cm}^{-1}$ was estimated to be $\sim 13 \mathrm{~cm}^{-1} .{ }^{24}$ The experimentally observed shifts are $10.1,9.4$, and $5.6 \mathrm{~cm}^{-1}$ for peaks a, b, and c, respectively. CASPT2 computed values are in excellent agreement at 9.3, 10.3, and 12.2 $\mathrm{cm}^{-1}$, with the largest deviation for $v\left(\mathrm{Cr}_{2}\right)$ where the effect of an elongated $\mathrm{Cr}-\mathrm{Cr}$ bond distance and anharmonicity of the mode is expected to be greatest. The aggregate $\mathrm{Cr}$ isotope shift from the three bands was $25.1 \mathrm{~cm}^{-1}$ in experiment and is $31.9 \mathrm{~cm}^{-1}$ with CASPT2.

Finally, we note that the largest complex, $\mathrm{Cr}_{2}(\mathrm{dmp})_{4}$, contains 78 atoms and the calculation was performed on 768 CPUs. The PHVA calculation took 5 days to complete involving 60 gradient calculations required for Hessian evaluation. FHVA would have required 468 gradient calculations.

Table 3: M06/def2-TZVP, PHVA CASPT2, and experimental $v\left(\mathrm{Cr}_{2}\right)$ in $\mathrm{cm}^{-1}$ are compared. The average value of all computed normal modes with significant $\mathrm{Cr}-\mathrm{Cr}$ motion are reported in parentheses.

\begin{tabular}{llll}
\hline Complex & M06 & CASPT2 & Experiment \\
\hline$\left[\mathrm{Cr}_{2}\left(\mathrm{O}_{2} \mathrm{CCH}_{3}\right)_{4}\right]\left(\mathrm{H}_{2} \mathrm{O}\right)_{2}$ & 585 & 196,266 & 150 to $250^{[a]}$ \\
$\mathrm{Cr}_{2}(\mathrm{mhp})_{4}$ & 607 & $282(299)$ & $556^{[b]}$ \\
& & & 340 or $400^{[c]}$ \\
$\mathrm{Cr}_{2}(\mathrm{dmp})_{4}$ & 623 & $310,324,353(329)$ & $345,363,387(365)^{[d]}$ \\
& & & $650^{[e]}$
\end{tabular}

[a] Ref 50; [b] Ref 11; [c] Ref 25; [d] Ref 24, Assigned as average of three bands. [e] Ref 24, Frequency estimated from aggregate isotope shift.

\section{Anharmonicity along the $\mathrm{Cr}-\mathrm{Cr}$ Stretching Mode}

Although the agreement between experiment and CASPT2 offers clear improvement over DFT, the bond distances are longer than what is observed for typical agreement for single-reference transitional metal complexes between DFT and experiment. Moreover, low frequency $\mathrm{Cr}-\mathrm{Cr}$ stretching modes are known to exhibit significant anharmonicity. ${ }^{11,24,25,42,44,50}$ Therefore, a scan of the PES along the harmonic normal mode was performed at the same level of theory as in the harmonic 
vibrational analysis and by including the so-called IPEA shift in the CASPT2 zeroth-order Hamiltonian. For $\mathrm{Cr}_{2}(\mathrm{mhp})_{4}$ and $\mathrm{Cr}_{2}(\mathrm{dmp})_{4}$, the structure was displaced along the harmonic mode associated with the $\mathrm{Cr}-\mathrm{Cr}$ stretching motion, $\mathrm{Q}_{i}$, in the positive and negative direction up to $3 \mathrm{Q}_{i}$. The resulting potential was fit to the fourth order polynomial,

$$
E\left(Q_{i}\right)=a Q_{i}^{2}+b Q_{i}^{3}+c Q_{i}^{4}
$$

to obtain cubic and quartic anharmonic corrections. This approach has been used in a series of $\mathrm{Fe}(\mathrm{II})$ spin-crossover complexes. ${ }^{51}$ The harmonic potential is included for comparison where $\mathrm{b}$ and $\mathrm{c}$ are set to zero and $\mathrm{a}=\frac{1}{2} \omega_{i}$ where $\omega_{i}$ is the harmonic vibrational frequency (Figure 4). Significant anharmonicity is observed in both complexes. The fitting results in an very large negative value for $\mathrm{b}$ due to the significant opening of the potential on the right hand side, as well as a large positive value for c corresponding to a symmetric loosening of the curve. The both fits have values of $R^{2}$ close to one and specific parameters obtained are $\mathrm{a}=135.86, \mathrm{~b}=-26.24$, and $\mathrm{c}=0.40$ for $\mathrm{Cr}_{2}(\mathrm{mhp})_{4}$ and $\mathrm{a}=166.43, \mathrm{~b}=-14.92$, and $\mathrm{c}=0.20$ for $\mathrm{Cr}_{2}(\mathrm{dmp})_{4}$. The anharmonic frequency can be obtained from the quadratic term and is $272 \mathrm{~cm}^{-1}$ for $\mathrm{Cr}_{2}(\mathrm{mhp})_{4}$ and $333 \mathrm{~cm}^{-1}$ for $\mathrm{Cr}_{2}(\mathrm{mhp})_{4}$. The inclusion of anharmonic corrections shifts the vibrational mode to a lower value compared to the harmonic mode by 10 and $20 \mathrm{~cm}^{-1}$, respectively. The CASPT2 minima obtained from the PES scan of $\mathrm{Cr}_{2}(\mathrm{mhp})_{4}$ without the IPEA shift is the same as that obtained by geometry optimization, $1.989 \AA$. This is true using either the Dunning or the ANO-RCC basis sets. However, the minima shifted to $1.887 \AA$ when the IPEA shift is employed, in excellent agreement with the experimental value of 1.889(1) $\AA$ (Table 4, Figures S3 and S4). These results are consistent with work by Ruiperez et al. on diatomic $\mathrm{Cr}_{2},{ }^{42}$ and the importance of both non-dynamical and dynamical correlation in accurately computing $\mathrm{Cr}-\mathrm{Cr}$ bond distances and vibrational modes. We note that an even larger IPEA shift of 0.45 was recommended for computing spectroscopic parameters in diatomic $\mathrm{Cr}_{2}$ by Ruiperez et al. While an analogous systematic study of different levels of theory is outside the scope of this work, one is necessary to justify the choice of computational model rigorously. 


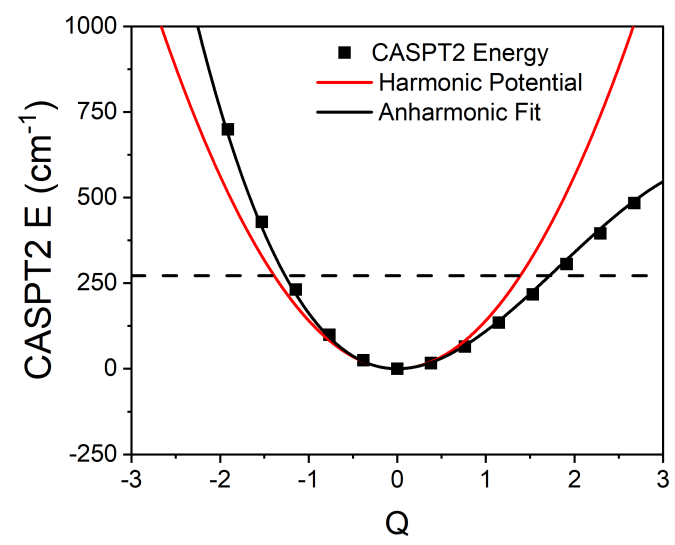

(a) $\mathrm{Cr}_{2}(\mathrm{mhp})_{4}$

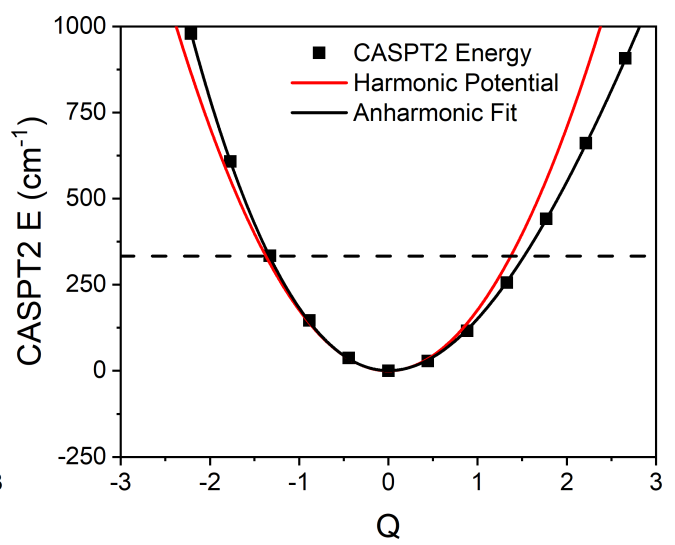

(b) $\mathrm{Cr}_{2}(\mathrm{dmp})_{4}$

Figure 4: The CASPT2 PES along the harmonic $\mathrm{Cr}-\mathrm{Cr}$ normal mode. On the left, the polynomial fit for $\mathrm{Cr}_{2}(\mathrm{mhp})_{4}\left(\mathrm{a}=135.86, \mathrm{~b}=-26.24, \mathrm{c}=0.40 ; \mathrm{R}^{2}=0.99966\right)$ is shown along with the harmonic potential for the mode at $282 \mathrm{~cm}^{-1}(\mathrm{a}=140.76, \mathrm{~b}=\mathrm{c}=0)$. On the right, the polynomial fit for $\mathrm{Cr}_{2}(\mathrm{dmp})_{4}\left(\mathrm{a}=166.43, \mathrm{~b}=-14.92, \mathrm{c}=0.20 ; \mathrm{R}^{2}=0.99996\right)$ is shown along with the harmonic potential for the mode at $282 \mathrm{~cm}^{-1}(\mathrm{a}=176.72, \mathrm{~b}=\mathrm{c}=0)$. The dashed black line denotes the predicted fundamental frequency.

Table 4: Effect of the so-called IPEA shift on $\mathrm{Cr}-\mathrm{Cr}$ bond distances. Values reported in $\AA$. The values for IPEA $=0.0$ are obtained by CASPT2 geometry optimizations while the values with IPEA $=0.25$ are obtained via normal mode sampling.

\begin{tabular}{lccc}
\hline Complex & $\begin{array}{c}\text { CASPT2/cc-pVTZ } \\
\text { IPEA }=0.0\end{array}$ & $\begin{array}{c}\text { CASPT2/ANO-RCC } \\
\text { IPEA }=0.25\end{array}$ & Exp. \\
\hline $\mathrm{Cr}_{2}(\mathrm{mhp})_{4}$ & 1.989 & 1.887 & $1.889(1)^{[a]}$ \\
$\mathrm{Cr}_{2}(\mathrm{dmp})_{4}$ & 1.922 & 1.850 & $1.849(2)^{[b]}$ \\
\hline
\end{tabular}

[a] Ref 11, [b] Ref 49

\section{Conclusions}

CASPT2 harmonic vibrational analysis can provide insight into the complex low-frequency region of experimental vibrational spectra for large dichromium complexes. The calculated geometries and vibrational modes are consistent with empirical $\mathrm{Cr}_{2}$ bond distances and frequencies. Specifically, the CASPT2 harmonic Cr-Cr stretching mode is predicted to lie at 196/266, 282, and 353 $\mathrm{cm}^{-1}$ for $\left[\mathrm{Cr}_{2}\left(\mathrm{O}_{2} \mathrm{CCH}_{3}\right)_{4}\right]\left(\mathrm{H}_{2} \mathrm{O}\right)_{2}, \mathrm{Cr}_{2}(\mathrm{mhp})_{4}$, and $\mathrm{Cr}_{2}(\mathrm{dmp})_{4}$, respectively. The bonding in the three complexes is weaker than their formal assignments based on bond distance and consistent with lower frequency vibrational modes. The complexity of the potential energy surface around the 
$\mathrm{Cr}$ centers manifests in this rich low-energy spectrum, in particular for $\mathrm{Cr}_{2}(\mathrm{mhp})_{4}$ and $\mathrm{Cr}_{2}(\mathrm{dmp})_{4}$. The modes between 250 and $400 \mathrm{~cm}^{-1}$ include normal modes that couple metal-metal and metalligand motions. While not all of these modes are confirmed to be Raman active, those that shift upon isotope substitution are consistent with the observed experimental bands, albeit systematically shifted to lower values. The PES calculations along the $v\left(\mathrm{Cr}_{2}\right)$ normal mode and observation of improved $\mathrm{Cr}-\mathrm{Cr}$ bond distances with the inclusion of the IPEA shift support the hypothesis by Da $\mathrm{Re}$ et al. that there is a distorted $\mathrm{Cr}-\mathrm{Cr}$ potential in these $\mathrm{Cr}(\mathrm{II})_{2}$ complexes similar to that inferred for $\mathrm{Cr}(0)_{2}$ contributing to the vibrational behavior of the $\mathrm{Cr}$ - $\mathrm{Cr}$ complexes. ${ }^{24}$ Finally, the $v\left(\mathrm{Cr}_{2}\right)$ mode is anharmonic and including cubic and quartic corrections shifts the vibrational mode

to lower values by 10 to $20 \mathrm{~cm}^{-1}$. Nevertheless, questions remain in describing this complicated low frequency region, in particular the nature of peaks assigned to an excited state transitions observed in the spectra, assumed to arise from the so-called $\delta \rightarrow \delta^{*}$ transition. These results emphasize that the interpretation of vibrational spectra for complexes with strong metal-metal and metal-ligand interactions is challenging requiring insights from both experiment and theory. CASPT2 vibrational analysis is anticipated to be a valuable tool in furthering understanding of multiconfigurational bonds in larger complexes with metal-metal bonds.

\section{Acknowledgements}

This work was supported by the Department of Energy, Office of Science, Basic Energy Sciences grant No. DE-SC0019463. Computations supporting this project were performed on High Performance Computing systems at the University of South Dakota, funded by NSF Award OAC1626516, and at the National Energy Research Scientific Computing Center (NERSC), a U.S. Department of Energy Office of Science User Facility operated under Contract No. DE-AC02$05 \mathrm{CH} 11231$. 


\section{Supporting Information}

Coordinates of optimized geometries, figures of active orbitals, tables with additional geometric parameters, CASSCF occupation numbers, relative energies, and additional computations of vibrational modes are provided as supporting information.

\section{References}

(1) Cotton, F. A.; Curtis, N. F.; Harris, C. B.; Lippard, S. J. Mononuclear and Polynuclear Chemistry of Rhenium (III): Its Pronouced Homophilicity. Inorg. Chem. 1964, 145, 1305-1307.

(2) Cotton, F. A.; Murillo, C. A.; Walton, R. A. Multiple Bonds Between Metal Atoms, 3rd ed.; Springer: Boston, Massachusettes, 2005; pp 35-68.

(3) Berry, J. F. Metal-Metal Bonds: From Fundamentals to Applications. Inorg. Chem. 2017, 56, $7577-7581$.

(4) Nguyen, T.; Sutton, A. D.; Brynda, M.; Fettinger, J. C.; Long, G. J.; Power, P. P. Synthesis of a stable compound with fivefold bonding between two chromium(I) centers. Science $\mathbf{2 0 0 5}$, $310,844-847$.

(5) Meyer, T. J.; Caspar, J. V. Photochemistry of metal-metal bonds. Chemical Reviews 1985, 85, $187-218$.

(6) McGrady, J. E. Molecular Metal-Metal Bonds; John Wiley \& Sons, Ltd, 2015; Chapter 1, pp $1-22$.

(7) Doherty, R. Meeting of the Metals. Nat. Chem. 2013, 5, 358-359.

(8) Introduction to Metal--Organic Frameworks. Chem. Rev. 2012, 112, 673-674, PMID: 22280456. 
(9) Chisholm, M.; Patmore, N. Molecular Metal-Metal Bonds: Compounds, Synthesis, Properties; Wiley-Blackwell: United States, 2015; pp 139-174.

(10) Alberding, B. G.; Chisholm, M. H.; Chou, Y.-H.; Gallucci, J. C.; Ghosh, Y.; Gustafson, T. L.; Patmore, N. J.; Reed, C. R.; Turro, C. Quadruply Bonded Dimetal Units Supported by 2,4,6Triisopropylbenzoates $\mathrm{MM}(\mathrm{TiPB})_{4}\left(\mathrm{MM}=\mathrm{Mo}_{2}\right.$, MoW, and $\left.\mathrm{W}_{2}\right)$ : Preparation and Photophysical Properties. Inorg. Chem. 2009, 48, 4394-4399.

(11) Cotton, F. A.; Fanwick, P. E.; Niswander, R. H.; Sekutowski, J. C. A triad of homologous, air-stable compounds containing short, quadruple bonds between metal atoms of Group 6. J. Am. Chem. Soc. 1978, 100, 4725-4732.

(12) Cotton, F. A.; Koch, S.; Millar, M. Exceedingly short metal-to-metal multiple bonds. J. Am. Chem. Soc. 1977, 99, 7372-7374.

(13) Kreisel, K. A.; Yap, G. P. A.; Dmitrenko, O.; Landis, C. R.; Theopold, K. H. The Shortest Metal-Metal Bond Yet: Molecular and Electronic Structure of a Dinuclear Chromium Diazadiene Complex. J. Am. Chem. Soc. 2007, 129, 14162-14163, PMID: 17967028.

(14) Noor, A.; Wagner, F. R.; Kempe, R. Metal-Metal Distances at the Limit: A Coordination Compound with an Ultrashort Chromium-Chromium Bond. Angew. Chem., Int. Ed. 2008, 47, 7246-7249.

(15) Eisenhart, R. J.; Carlson, R. K.; Boyle, K. M.; Gagliardi, L.; Lu, C. C. Synthesis and redox reactivity of a phosphine-ligated dichromium paddlewheel. Inorganica Chim. Acta 2015, 424, 336-344, Metal-Metal Bonded Compounds and Metal Clusters.

(16) Huang, Y.-L.; Lu, D.-Y.; Yu, H.-C.; Yu, J.-S. K.; Hsu, C.-W.; Kuo, T.-S.; Lee, G.-H.; Wang, Y.; Tsai, Y.-C. Stepwise Construction of the Cr-Cr Quintuple Bond and its Destruction upon Axial Coordination. Angew. Chem., Int. Ed. 2012, 51, 7781-7785. 
(17) Ketkar, S. N.; Fink, M. Structure of dichromium tetraacetate by gas-phase electron diffraction. J. Am. Chem. Soc. 1985, 107, 338-340.

(18) Cotton, F. A.; Hillard, E. A.; Murillo, C. A.; Zhou, H.-C. After 155 Years, A Crystalline Chromium Carboxylate with a Supershort Cr-Cr Bond. J. Am. Chem. Soc. 2000, 122, 416417.

(19) La Macchia, G.; Li Manni, G.; Todorova, T. K.; Brynda, M.; Aquilante, F.; Roos, B. O.; Gagliardi, L. On the Analysis of the Cr-Cr Multiple Bond in Several Classes of Dichromium Compounds. Inorg. Chem. 2010, 49, 5216-5222, PMID: 20426405.

(20) Li Manni, G.; Dzubak, A. L.; Mulla, A.; Brogden, D. W.; Berry, J. F.; Gagliardi, L. Assessing Metal-Metal Multiple Bonds in Cr-Cr, Mo-Mo, and W-W Compounds and a Hypothetical U-U Compound: A Quantum Chemical Study Comparing DFT and Multireference Methods. Chem. Eur. J. 2012, 18, 1737-1749.

(21) Huang, G.-T.; Yu, J.-S. K. Analyses on Molecular Properties of the Diamidinate CrI-CrI Complex by Multireference and DFT Approaches. J. Phys. Chem. A 2019, 123, 7803-7813, PMID: 31424207.

(22) Spivak, M.; López, X.; de Graaf, C. Trends in the Bond Multiplicity of Cr2, Cr3, and Cr2M $(\mathrm{M}=\mathrm{Zn}, \mathrm{Ni}, \mathrm{Fe}, \mathrm{Mn})$ Complexes Extracted from Multiconfigurational Wave Functions. $J$. Phys. Chem. A 2019, 123, 1538-1547.

(23) Ndambuki, S.; Ziegler, T. A Theoretical Analysis of Supported Quintuple and Quadruple Chromium-Chromium Bonds. Inorg. Chem. 2013, 52, 3860-3869, PMID: 23480651.

(24) Da Re, R. E.; Eglin, J. L.; Carlson, C. N.; John, K. D.; Morris, D. E.; Woodruff, W. H.; Bailey, J. A.; Batista, E.; Martin, R. L.; Cotton, F. A.; Hillard, E. A.; Murillo, C. A.; Sattelberger, A. P.; Donohoe, R. J. Nature of Bonding in Complexes Containing "Supershort" Metal-Metal Bonds. Raman and Theoretical Study of M2(dmp)4 [M = Cr (Natural Abun- 
dance $\mathrm{Cr}$, $50 \mathrm{Cr}$, and $54 \mathrm{Cr}$ ) and $\mathrm{Mo}$; dmp = 2,6-Dimethoxyphenyl]. J. Am. Chem. Soc. 2010, 132, 1839-1847, PMID: 20092271.

(25) Manning, M. C.; Trogler, W. C. Electronic absorption and emission spectra of complexes containing dichromium, dimolybdenum, and ditungsten quadruple bonds. J. Am. Chem. Soc. 1983, 105, 5311-5320.

(26) Park, J. W.; Al-Saadon, R. R.; MacLeod, M. K.; Shiozaki, T.; Vlaisavljevich, B. Multireference Electron Correlation Methods: Journeys Along Potential Energy Surfaces. Chem. Rev. 2020, 120, 5878-5909.

(27) MacLeod, M. K.; Shiozaki, T. Automatic Code Generation Enables Nuclear Gradient Computations for Fully Internally Contracted Multireference Theory. J. Chem. Phys. 2015, 142, 051103 .

(28) Vlaisavljevich, B.; Shiozaki, T. Nuclear Energy Gradients for Internally Contracted Complete Active Space Second-Order Perturbation Theory: Multistate Extensions. J. Chem. Theory Comput. 2016, 12, 3781-3787.

(29) Shiozaki T.; Vlaisavljevich B. Computational Spectroscopy of the Cr-Cr Bond in Coordination Complexes. ChemRxiv. Cambridge: Cambridge Open Engage; 2021; This content is a preprint and has not been peer-reviewed.

(30) Li, H.; Jensen, J. H. Partial Hessian Vibrational Analysis: The Localization of the Molecular Vibrational Energy and Entropy. Theo. Chem. Acc. 2002, 107, 211-219.

(31) Jin, S.; Head, J. D. Theoretical investigation of molecular water adsorption on the $\mathrm{Al}(111)$ surface. Surf. Sci. 1994, 318, 204-216.

(32) Calvin, M. D.; Head, J. D.; Jin, S. Theoretically modelling the water bilayer on the Al(111) surface using cluster calculations. Surf. Sci. 1996, 345, 161-172. 
(33) Head, J. D. Computation of vibrational frequencies for adsorbates on surfaces. Int. J. Quant. Chem. 1997, 65, 827-838.

(34) Head, J. D.; Shi, Y. Characterization of Fermi resonances in adsorbate vibrational spectra using cluster calculations: Methoxy adsorption on $\mathrm{Al}(111)$ and $\mathrm{Cu}(111)$. International Journal of Quantum Chemistry 1999, 75, 815-820.

(35) Head, J. D. A vibrational analysis with Fermi resonances for methoxy adsorption on $\mathrm{Cu}(111)$ using ab initio cluster calculations. International Journal of Quantum Chemistry 2000, 77, $350-357$.

(36) BAGEL, Brilliantly Advanced General Electronic-structure Library. http://www.nubakery.org under the GNU General Public License.

(37) Dunning, T. H. Gaussian basis sets for use in correlated molecular calculations. I. The atoms boron through neon and hydrogen. J. Chem. Phys. 1989, 90, 1007-1023.

(38) Balabanov, N. B.; Peterson, K. A. Systematically convergent basis sets for transition metals. I. All-electron correlation consistent basis sets for the 3d elements Sc-Zn. J. Chem. Phys. 2005, 123, 064107.

(39) Balabanov, N. B.; Peterson, K. A. Basis set limit electronic excitation energies, ionization potentials, and electron affinities for the $3 \mathrm{~d}$ transition metal atoms: Coupled cluster and multireference methods. J. Chem. Phys. 2006, 125, 074110.

(40) Weigend, F. Accurate Coulomb-fitting basis sets for H to Rn. Phys. Chem. Chem. Phys. 2006, $8,1057-1065$.

(41) Ghigo, G.; Roos, B. O.; Åke Malmqvist, P. A modified definition of the zeroth-order Hamiltonian in multiconfigurational perturbation theory (CASPT2). Chem. Phys. Lett. 2004, 396, $142-149$. 
(42) Ruipérez, F.; Aquilante, F.; Ugalde, J. M.; Infante, I. Complete vs Restricted Active Space Perturbation Theory Calculation of the Cr2 Potential Energy Surface. Journal of Chemical Theory and Computation 2011, 7, 1640-1646.

(43) Zobel, J. P.; Nogueira, J. J.; González, L. The IPEA dilemma in CASPT2. Chem. Sci. 2017, 8, 1482-1499.

(44) Roos, B. O.; Andersson, K. Multiconfigurational Perturbation Theory with Level Shift - The $\mathrm{Cr}_{2}$ Potential Revisited. Chem. Phys. Lett. 1995, 245, 215-223.

(45) Ahlrichs, R. et al. TURвомоLe, version 7.3, Universität Karlsruhe. 2018.

(46) Zhao, Y.; Truhlar, D. G. The M06 suite of density functionals for main group thermochemistry, thermochemical kinetics, noncovalent interactions, excited states, and transition elements: Two new functionals and systematic testing of four M06-class functionals and 12 other functionals. Theor. Chem. Acc. 2008, 120, 215-241.

(47) Weigend, F.; Ahlrichs, R. Balanced basis sets of split valence, triple zeta valence and quadruple zeta valence quality for H to Rn: Design and assessment of accuracy. Phys. Chem. Chem. Phys. 2005, 7, 3297-3305.

(48) Cotton, F. A.; Deboer, B. G.; Laprade, M. D.; Pipal, J. R.; Ucko, D. A. Multiple Chromium(II)-Chromium(II) and Rhodium(II)-Rhodium(II) Bonds. J. Am. Chem. Soc. 1970, 92, 2926-2927.

(49) Cotton, F. A.; Koch, S. A.; Millar, M. Dichromium and dimolybdenum compounds of 2,6dimethoxyphenyl and 2,4,6-trimethoxyphenyl ligands. Inorg. Chem. 1978, 17, 2087-2093.

(50) Rice, S. F.; Wilson, R. B.; Solomon, E. I. Electronic absorption spectrum of chromous acetate dihydrate and related binuclear chromous carboxylates. Inorg. Chem. 1980, 19, 3425-3431.

(51) Wu, J.; Sousa, C.; de Graaf, C. The Role of Vibrational Anharmonicity in the Computational Study of Thermal Spi Crossover. Magnetochemistry 2019, 5, 49. 


\section{Graphical TOC Entry}

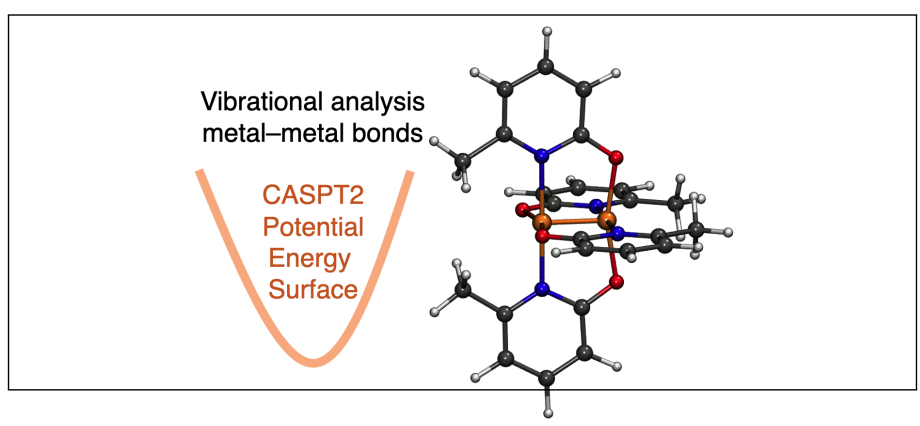

Synopsis: The computational vibrational spectrum of $\mathrm{Cr}-\mathrm{Cr}$ bonds in coordination complexes can be modeled using multireference complete active space method (CASPT2), allowing direct comparison with experimental spectroscopic data. The computation of the Hessian by gradient finite difference and the resulting spectra for three complexes are reported. 